\title{
Association Between Salivary Carbonic Anhydrase 6 Levels and Early Childhood Caries in Children With Chronic Bronchial Asthma
}

\section{Essa Ahmed Kormi}

Security Forces Hospital

Sharat Chandra Pani ( $\sim$ spani@uwo.ca )

Western University Schulich School of Medicine and Dentistry https://orcid.org/0000-0002-5052-5986

Reem AlNatsha

Security Forces Hospital

Research article

Keywords: Carbonic Anhydrase 6, Bronchial Asthma, Dental Caries

Posted Date: December 17th, 2020

DOI: https://doi.org/10.21203/rs.3.rs-129150/v1

License: (c) (i) This work is licensed under a Creative Commons Attribution 4.0 International License.

Read Full License 


\section{Abstract}

Background and Aim: The aim of this study was to compare the level of a salivary marker (carbonic anhydrase 6 isoenzyme (CA6)) between children with and without bronchial asthma (BA) with dental caries to age and gender-matched non-asthmatic children with a similar caries profile.

Materials and Methods: Saliva samples were collected from 80 children aged 6 to 8 years. The children were divided into four groups according to the presence of BA and caries: 1) high caries, presence of $B A$, 2) absence of caries, presence of $B A, 3$ ) high caries, absence of $B A, 4)$ absence of caries, absence of BA . Differences in CA6 were compared among the groups using a One-Way ANOVA and Scheffe's post hoc test. Separate regression models were developed to evaluate the impact of oral hygiene, dental caries and salivary $\mathrm{pH}$ on $\mathrm{CA} 6$ levels in children with and without BA.

Results: Children with dental caries had a significantly higher CA6 level than their caries free healthy controls. Children with BA and dental caries had a significantly lower CA6 level than their caries free counterparts. The linear regression model showed dental caries had a significant inverse association with CA6 expression in children with BA but not in healthy children.

Conclusion: CA6 may serve as a protective mechanism against dental caries in children with bronchial asthma.

\section{Introduction}

Bronchial Asthma (BA) can be defined as inflammation of the airways that leads to intermittent bronchospasms characterized by bouts of coughing, wheezing, chest tightness, dyspnea along with the variable degree of expiratory airflow obstruction(1). The prevalence of BA around the world is estimated to be around 300 million at present, with children being more affected than adults.(1-3) The treatment of BA includes medications such as B2 agonists and steroids that can adversely affect the oral health of patients by causing xerostomia and altering the composition of saliva. $(2,4,5)$

Dental caries is a multifactorial disease that can be influenced by salivary composition, nature of diet consumed, cultural aspects and also the genetic nature of the tooth itself.(6) Studies have suggested that children with asthma are at a higher risk for dental caries than non-asthmatic children. $(4,5)$ While the role of medications inthe reduction of salivary flow have been suggested as the reason for this increased risk, there are studies that have suggested that there may be an alteration of underlying salivary defense mechanisms in children with BA. $(4,5,7,8)$

Of the many salivary defense mechanisms, the salivary buffering capacity is particularly effective in neutralizing acid production by cariogenic bacteria. $(7,9,10)$ There are three buffering mechanisms in saliva; the phosphate, the protein buffer and the carbonic acid-bicarbonate buffer(11). The carbonic acidbicarbonate buffer is the major buffer in stimulated saliva, and arguably the most important in reducing caries risk. (10-13)The rate limiting step of this buffer is regulated by the enzyme Carbonic carbonic 
anhydrase (CA), of which the isoenzyme carbonic anhydrase 6 (CA6) is the only one found in saliva and is secreted by the acinar cells of the submandibular gland.(13) CA6 activity is used as indicator of the function of the carbonic acid-bicarbonate buffer system, with studies showing increased CA6 activity in the saliva and plaque of healthy children with high levels of dental caries. $(14,15)$ In children with BA it has been suggested that there may be increase in $\mathrm{CA}$ isoenzymes as there is a proven association between the increased CA levels and the cellular activation of eosinophils which is the hallmark of eosinophilia $(16,17)$. However, there is little known about the effects of dental caries on the CA6 levels of children with BA..

The primary aim of this study was to compare the level of salivary CA6 isoenzyme in children with BA having dental caries to age and gender-matched non-asthmatic children with a similar caries profile. The study also aimed to study the CA6 profile of caries-free children with BA to that of caries-free healthy children.

\section{Materials And Methods}

The study was approved in the research center of the Riyadh Elm University (FPGRP/43735004/277). Informed consent was obtained from the parents of children before saliva collection and verbal consents were obtained from the participating children before the collection of saliva was used in the study. Confidentiality of the participant was maintained throughout the study.

A sample power calculation was performed using the G-power sample power calculator ver. 3.1 ( Universtat Kiel, Kiel, Germany). It was estimated that for a power of 0.95 , with an effect size of 0.8 a minimum of 20 children per group was needed to perform a One-Way ANOVA.

Children with BA were selected from the pediatric clinics of the Security Forces Hospital, Riyadh Saudi Arabia.). The inclusion criteria were that the participants were aged 6-8 years with bronchial asthma and (for group 1) childhood caries. Participants who were free of BA were selected from the dental clinics of Riyadh Elm University. The control and experimental groups were matched with each other according to age, gender, and extent of dental problems. A total of four groups were divided according to caries:

Group 1: The participants in this group had 4 or more dental caries lesions in the primary teeth (with or without dental caries on the first permanent molar); had at least one asthmatic attack in the past three months and were currently on Albuterol, Salbutamol or Terbutaline but were not on any other medication.

Group 2: The participants in this group were free of any dental caries and had at least one episode of asthmatic attack in the past three months. They were currently on Albuterol, Salbutamol or Terbutaline but were not on any other medication.

Group 3: The participants of this group had four or more active dental caries lesions in the primary teeth (with or without dental caries on the first permanent molar) and had no history of BA.

Group 4: The participants in this group were free of any dental caries and had no history of BA 
Dental caries profile of the primary teeth was measured using the decayed filled teeth index (dft) and the dental caries profile of the permanent was measured using the Decayed, Missing Filled permanent teeth index (DMFT). Oral hygiene was evaluated using the oral hygiene index-simplified (OHI-S). The teeth were examined using WHO category II criteria - clinical examination with dental unit light and without radiographs. Data collection was conducted by one examiner and one data recorder (EK).

Saliva was allowed to pool in the floor of the mouth by asking the child to touch the chin to the chest for approximately one minute. Saliva was then collected using a sterile Pasteur pipette and transferred to a $1.5 \mathrm{ml}$ Eppendorf tube. These sterile plastic collection tubes were closed carefully and placed in a 2-80C ice storage box to be transferred to the deep freezer of a $\triangle 600 \mathrm{C}$ until they could be analyzed. CA6 assays were performed using the zymographic method using a Human CA6 ELISA kit (Abbexa Ltd, Cambridge Science Park, and Cambridge, CB4 OEY, UK).(14)

The Shapiro Wilk test of CA6 levels showed a Kurtosis of 0.456 and skew of -0.01 , suggesting that the sample was normally distributed. Therefore, parametric statistics were used. The difference in salivary carbonic anhydrase between the test and control groups was measured using the one-way analysis of variance (ANOVA) and Scheffe's post hoc test A linear regression model using the CA-VI levels; a dependent variable and gender, caries and asthmatic medication as independent variables were designed. All Statistical analyses were performed using the statistical package for social sciences (SPSS) 25 data management software (IBM SPSS, Armonk, NY, USA).

\section{Results}

The sample comprised of 80 subjects aged between 6 to 8 years with a mean age of 6.77 years (SD +/2.1). There were 46 males and 34 females and although the mean age of the females was greater than that of the males the differences were not statistically significant $(p=0.670)$. When the oral hygiene scores among the groups were compared it was observed that children with dental caries had significantly higher $\mathrm{OHI}(\mathrm{S})$ scores compared to children who were caries free. This was true of both children with and without BA. (Table 1)

Table 1. Comparison of Oral Hygiene Index Scores among groups.

\begin{tabular}{|c|c|c|c|c|c|}
\hline & $\mathbf{N}$ & Mean & Std. Deviation & $F^{*}$ & Sig** \\
\hline Healthy High Caries ${ }^{a}$ & 20 & 2.040 & 0.66 & 7.760 & $0.001^{\star \star}$ \\
\hline Healthy Caries Free ${ }^{b}$ & 20 & 1.414 & 0.52 & & \\
\hline Asthma High ${ }^{a}$ Caries & 20 & 2.240 & 0.89 & & \\
\hline Asthma Caries Free ${ }^{b}$ & 20 & 1.291 & 0.82 & & \\
\hline
\end{tabular}


* Calculated using the One-Way ANOVA; ** differences significant at $p<0.05$; $a$, b: Differences in superscript indicate significant inter group differences $(p<0.05)$ when using the Scheffe's post hoc test.

When the CA6 levels of the three groups were compared it was observed that significant difference existed among the four groups studied (Table 2). The Scheffe's post hoc test showed that CA6 of Asthmatic Children without dental caries was significantly lower than healthy children with dental caries, healthy children who were caries free and Asthmatic children with dental caries. Healthy children who had high caries had a significantly higher CA6 groups when compared to the other groups. Children with BA who were caries free had lower salivary CA6 levels when compared to their healthy caries controls but these differences were not statistically significant (Table 2).

Table 2. Comparison of CAVI levels among different groups.

\begin{tabular}{|c|c|c|c|c|}
\hline & $\begin{array}{l}\text { Mean CA6 } \\
\text { (pcg/ml) }\end{array}$ & Std. Deviation & $F^{*}$ & Sig** \\
\hline Healthy High Caries ${ }^{a}$ & 21.620 & 3.09 & 265.123 & $<0.001$ * \\
\hline Healthy Caries Free ${ }^{b}$ & 14.115 & 2.58 & & \\
\hline Asthma High Caries ${ }^{c}$ & 7.030 & 1.17 & & \\
\hline Asthma Caries Free ${ }^{b}$ & 12.270 & 1.18 & & \\
\hline
\end{tabular}

* Calculated using the One-Way ANOVA; ** differences significant at $\mathrm{p}<0.05 ; \mathrm{a}$, b: Differences in superscript indicate significant inter group differences $(p<0.05)$ when using the Scheffe's post hoc test.

Table 3. Regression model for factors influencing the expression of CA6 in Healthy patients and Asthmatic patients 


\begin{tabular}{|c|c|c|c|c|c|c|}
\hline \multirow[t]{2}{*}{ Model } & & \multicolumn{2}{|c|}{$\begin{array}{l}\text { Unstandardized } \\
\text { Coefficients }\end{array}$} & \multirow{2}{*}{$\begin{array}{l}\text { Standardized } \\
\text { Coefficients } \\
\text { Beta }\end{array}$} & \multirow[t]{2}{*}{$\mathrm{t}$} & \multirow[t]{2}{*}{ Sig. } \\
\hline & & B & Std. Error & & & \\
\hline \multirow{5}{*}{$\begin{array}{l}\text { Bronchial } \\
\text { Asthma }\end{array}$} & (Constant) & 14.841 & 4.509 & & 3.292 & .002 \\
\hline & Sex & -1.104 & .663 & -.188 & -1.666 & .105 \\
\hline & $\mathrm{OHI}$ & .325 & .408 & .110 & .797 & .431 \\
\hline & $\begin{array}{l}\text { Total number of } \\
\text { decayed teeth }\end{array}$ & -3.658 & .117 & -.928 & -5.641 & .000 \\
\hline & Saliva $\mathrm{pH}$ & -.142 & .457 & -.043 & -.311 & .758 \\
\hline \multirow{5}{*}{$\begin{array}{l}\text { No-Bronchial } \\
\text { Asthma }\end{array}$} & (Constant) & 16.790 & 9.154 & & 1.288 & .001 \\
\hline & Sex & -1.104 & .663 & -.188 & -1.666 & .105 \\
\hline & $\mathrm{OHI}$ & 4.447 & 1.079 & .062 & .414 & .006 \\
\hline & $\begin{array}{l}\text { Number of Decayed } \\
\text { teeth }\end{array}$ & 1.619 & 6.733 & -.053 & -.389 & .699 \\
\hline & Saliva pH & 3.224 & 1.049 & .032 & .213 & .025 \\
\hline
\end{tabular}

a. Dependent Variable: CA6.

Separate linear regression models with CA6 levels as the dependent variable were developed for patients with and without BA. In patients with BA it was found that there was a significant negative association between CA6 and the total number of decayed teeth (Table 3). When a similar model was constructed for the control group it was observed that while $\mathrm{OHI}$ and salivary $\mathrm{pH}$ had a significant positive association with dental caries. Although there was a positive association between levels of CA6 and number of decayed teeth, the association was not statistically significant (Table 3).

\section{Discussion}

CA6 is the only isoenzyme of the CA family that is secreted in the saliva $(13,15,18)$. While there is evidence to suggest that an increased CA6 level is an indicator of caries activity (15), there is also evidence to suggest that impaired CA6 activity may cause dental caries.(18) The current study sought to explore the impact of bronchial asthma (BA) on the CA6 mechanism in the oral cavity and its relationship to dental caries.

While dental caries is multifactorial and not limited to a single biomarker, in conditions such as bronchial asthma, where there is a definite challenge to oral health, the identification of certain protective enzyme pathways could greatly influence future preventive dental research.(11) Increased dental caries in BA has 
been recognized for over 30 years.(19) Whether this is due to the effects of medication causing xerostomia, or whether there is a deeper physiological reason remains a point of debate. $(4,5,20)$ The fact that children with BA who had dental caries showed a decrease in CA6 levels in contrast to healthy children with dental caries who showed increased CA6 levels lends credence to the hypothesis that BA may alter salivary buffering capacity. The finding may also explain the seeming contradiction between an increased CA6 level in children with dental caries and decreased CA6 levels reported in severe dental caries. $(12,15,18)$

The results of our study found that children with BA had significantly lower CA6 levels than their healthy counterparts. This is an interesting finding because it has been shown that that Carbonic carbonic Anhydrases anhydrase are is expressed in children with inflammatory conditions. $(17,21)$ The children with BA and dental caries had significantly lower CA6 levels when compared to their caries free counterparts. This is in contrast to healthy children where children with dental caries had significantly higher levels of CA6 than their caries free counterparts.

CA6 is a marker of the activity of the bicarbonate buffer in saliva.(12) The results of this study show that there was a significant association between salivary $\mathrm{pH}$ and dental caries among healthy children but not in the children with BA. While this supports the fact that children with BA may have a compromised salivary buffer, further research is needed to prove the actual mechanism by which the salivary CA6 mechanism is compromised. While the cellular inflammation and elevated eosinophilia of BA has been associated with increased levels of plasma CA isoenzymes such as Carbonic carbonic Anhydraseanhydrase-4 (CA4)(21), there is little known in literature about the impact of BA on salivary CA6.

The fact that asthmatic children who were caries free had a significantly higher expression of CA6 than their counterparts with dental caries is a significant factor. Asthma is a chronic airway disease characterized by inflammation and airway obstruction with bronchospasm.(1) When comparing the effects of dental caries on CA6 levels, the regression models seem to suggest that the impact of CA6 levels are more significant in children with BA than healthy controls. This seems to suggest that the function of the salivary CA6 pathway is probably critical to preventing dental caries in children with BA.

The findings of this study need to be viewed keeping in mind certain limitations. The main limitation of the study is that there could be several confounding factors such as dietary habits, socioeconomic status or fluoride adequacy that the study was unable to control for. The study was also unable to match for medication being used or possible salivary gland problems. It has been shown that failure to account for these confounders means that results should be interpreted with caution. $(22,23)$ While the findings of the study point to significant associations between CA6 and dental caries in children with bronchial asthma, the design of the study does allow for the assumption of causation. Another limitation of the study is that while the CA6 levels were measured, the study did not seek to measure bicarbonate ion concentration or $\mathrm{pH}$.The mechanism of salivary CA6 and the exact impact of BA on the enzyme pathway is also beyond the scope of the current study and would require further study. Despite these limitations, 
the results of this study highlight the oral health challenges faced by children with BA and support the need for the creation of multidisciplinary teams to manage the oral health of children with systemic disorders. (24)

\section{Conclusion}

There is a significantly lower expression of CA6 levels in children with bronchial asthma who have dental caries than those who do not have dental caries. While tThere is a significant negative association between the CA6 level and the number of decayed teeth in children with BA.

\section{Declarations}

Ethics approval and consent to participate: Ethical Approval was obtained from the research center of the Riyadh Elm University and all parents provided informed consent for the participations of their children

Consent for publication: Not applicable

Competing interests: The authors declare that they have no competing interests

Funding: The study was self funded

Authors' contributions: SCP designed the study and was responsible for statistical analyses, EaK was responsible for data collection and data analyses, RN was responsible for overall logistical management, data collection and data analyses. All authors contributed equally to the preparation of the manuscript

Data Availability: Data will be made available by the authors upon reasonable request

\section{References}

1. Becker AB, Abrams EM. Asthma guidelines: the Global Initiative for Asthma in relation to national guidelines. Curr Opin Allergy Clin Immunol. 2017 Apr;17(2):99-103.

2. Alaki SM, Ashiry EA, Bakry NS, Baghlaf KK, Bagher SM. The effects of asthma and asthma medication on dental caries and salivary characteristics in children. Oral Health Prev Dent. 2013;11(2):113-20.

3. Al-Malik MI, Rehbini YA. Prevalence of dental caries, severity, and pattern in age 6 to 7-year-old children in a selected community in Saudi Arabia. J Contemp Dent Pract. 2006 May;7(2):46-54.

4. Chellaih P, Sivadas G, Chintu S, Vaishnavi Vedam VK, Arunachalam R, Sarsu M. Effect of antiasthmatic drugs on dental health: A comparative study. J Pharm Bioallied Sci. 2016 Oct;8(Suppl 1):S77-80.

5. Ersin NK, Gülen F, Eronat N, Cogulu D, Demir E, Tanaç R, et al. Oral and dental manifestations of young asthmatics related to medication, severity and duration of condition. Pediatr Int. 2006 Dec;48(6):549-54. 
6. Kidd E, Fejerskov O. Changing concepts in cariology: forty years on. Dent Update. 2013 May;40(4):277-278,280-282,285-286.

7. Ryberg M, Möller $C$, Ericson T. Effect of beta 2-adrenoceptor agonists on saliva proteins and dental caries in asthmatic children. J Dent Res. 1987 Aug;66(8):1404-6.

8. Schmier JK, Manjunath R, Halpern MT, Jones ML, Thompson K, Diette GB. The impact of inadequately controlled asthma in urban children on quality of life and productivity. Ann allergy, asthma Immunol Off Publ Am Coll Allergy, Asthma, Immunol. 2007 Mar;98(3):245-51.

9. Garrett JR. The proper role of nerves in salivary secretion: a review. J Dent Res. 1987 Feb;66(2):38797.

10. Szabó I. Carbonic anhydrase activity in the saliva of children and its relation to caries activity. Caries Res. 1974;8(2):187-91.

11. Gao X, Jiang S, Koh D, Hsu C-YS. Salivary biomarkers for dental caries. Periodontol 2000. 2016 Feb;70(1):128-41.

12. Kimoto $\mathrm{M}$, Kishino $\mathrm{M}$, Yura $\mathrm{Y}$, Ogawa $\mathrm{Y}$. A role of salivary carbonic anhydrase $\mathrm{VI}$ in dental plaque. Arch Oral Biol. 2006 Feb;51(2):117-22.

13. Kivela J, Parkkila S, Parkkila AK, Leinonen J, Rajaniemi H. Salivary carbonic anhydrase isoenzyme VI. J Physiol. 1999 Oct;520 Pt 2(Pt 2):315-20.

14. Leinonen J, Kivelä J, Parkkila S, Parkkila AK, Rajaniemi H. Salivary carbonic anhydrase isoenzyme VI is located in the human enamel pellicle. Caries Res. 1999;33(3):185-90.

15. Picco D de CR, Lopes LM, Rocha Marques M, Line SRP, Parisotto TM, Nobre Dos Santos M. Children with a Higher Activity of Carbonic Anhydrase VI in Saliva Are More Likely to Develop Dental Caries. Caries Res. 2017;51(4):394-401.

16. Maric J, Ravindran A, Mazzurana L, Van Acker A, Rao A, Kokkinou E, et al. Cytokine-induced endogenous production of prostaglandin $D(2)$ is essential for human group 2 innate lymphoid cell activation. J Allergy Clin Immunol. 2019 Jun;143(6):2202-2214.e5.

17. Henry EK, Sy CB, Inclan-Rico JM, Espinosa V, Ghanny SS, Dwyer DF, et al. Carbonic anhydrase enzymes regulate mast cell-mediated inflammation. J Exp Med. 2016 Aug;213(9):1663-73.

18. Kivelä J, Parkkila S, Parkkila AK, Rajaniemi H. A low concentration of carbonic anhydrase isoenzyme VI in whole saliva is associated with caries prevalence. Caries Res. 1999;33(3):178-84.

19. Botelho MPJ, Maciel SM, Cerci Neto A, Dezan CC, Fernandes KBP, de Andrade FB. Cariogenic microorganisms and oral conditions in asthmatic children. Caries Res. 2011;45(4):386-92.

20. Putney JWJ. Identification of cellular activation mechanisms associated with salivary secretion. Annu Rev Physiol. 1986;48:75-88.

21. Wen T, Mingler MK, Wahl B, Khorki ME, Pabst O, Zimmermann N, et al. Carbonic anhydrase IV is expressed on IL-5-activated murine eosinophils. J Immunol. 2014 Jun;192(12):5481-9.

22. Patini R, Staderini E, Camodeca A, Guglielmi F, Gallenzi P. Case Reports in Pediatric Dentistry Journals: A Systematic Review about Their Effect on Impact Factor and Future Investigations. Dent J 
(Basel). 2019 Oct 24;7(4).

23. Romandini M, Gioco G, Perfetti G, Deli G, Staderini E, Laforì A. The association between periodontitis and sleep duration. J Clin Periodontol. 2017 May;44(5):490-501

24. Patini R, Staderini E, Gallenzi P. Multidisciplinary surgical management of Cowden syndrome: Report of a case. J Clin Exp Dent. 2016 Oct 1;8(4):e472-e474. 